\title{
Effect of Different Levels of Zeolite and Nitrogen on Grain Yield and Nutrient Uptake of Maize Grown in Red Soil
}

\author{
CH. Ravali*, K. Jeevan Rao, M. Srilatha and K. Suresh \\ PJTSAU, Rajendranagar, Hyderabad, 500030, India \\ *Corresponding author
}

\section{A B S T R A C T}

\section{Keywords}

Maize, Zeolite, Nitrogen, Grain yield, and $\mathrm{N}, \mathrm{P}, \mathrm{K}$ uptake

Article Info

Accepted: 04 May 2019 Available Online: 10 June 2019
The present study entitled "Influence of zeolite and nitrogen levels on grain yield and nutrient uptake of maize grown in red soils," was a pot culture study carried out in Green House of Soil Science department, College of Agriculture, Rajendranagar, PJTSAU, Hyderabad, Telangana state during kharif, 2018-19. The treatments consists of combinations of 3 levels of nitrogen $\left(100,150,200 \mathrm{~kg} \mathrm{ha}^{-1}\right)$ and 4 levels of zeolite $(0,2.5$, $5,7.5 \mathrm{t} \mathrm{ha}^{-1}$ ) along with a control in which only $\mathrm{P}$ and $\mathrm{K}$ were applied and they were replicated thrice in a factorial completely randomized design. The results revealed that application of $200 \mathrm{~kg} \mathrm{ha}^{-1}$ nitrogen in combination with $7.5 \mathrm{t} \mathrm{ha}^{-1}$ zeolite $\left(\mathrm{Z}_{7.5} \mathrm{~N}_{200}\right)$ increased grain yield of maize which was on par with the treatment receiving $200 \mathrm{~kg} \mathrm{ha}^{-1}$ nitrogen in combination with $5 \mathrm{t} \mathrm{ha}^{-1}$ zeolite $\left(\mathrm{Z}_{5.0} \mathrm{~N}_{200}\right)$. The combination of $7.5 \mathrm{t} \mathrm{ha}^{-1}$ zeolite with $200 \mathrm{~kg} \mathrm{ha}^{-1}$ nitrogen $\left(\mathrm{Z}_{7.5} \mathrm{~N}_{200}\right)$ have significantly increased $\mathrm{N}, \mathrm{P}, \mathrm{K}$ uptake of maize compared to rest of the treatments.

\section{Introduction}

Zeolites are among the most common minerals present in sedimentary rocks. They are natural crystalline tectosilicates (Szerement et al., 2014) exhibiting an open three-dimensional structure containing cations needed to balance the electrostatic charge of the framework of silica and alumina tetrahedral units. Pores and voids are the key characteristics of zeolite materials (Ramesh et al., 2011). Amendment of clinoptilolite zeolite to sandy soils has been reported to lower nitrogen concentration in the leachate and to increase moisture and nutrients in the soil due to increased soil surface area and cation exchange capacity (He et al., 2002). So, in order to improve nitrogen uptake in maize, zeolite can be used along with inorganic fertilizers.

Clinoptilolite promote better plant growth by improving the use efficiency of fertilizers due to its high adsorption rate, cation exchange, catalysis and dehydration capacities. It has a very high CEC (from 100 to $230 \mathrm{cmol} \mathrm{kg}^{-1}$ ). Therefore, its application to the soil increases the CEC of soils 2-3 times greater than other types of minerals found in soils. Keeping this in view, the mix of zeolite $(\mathrm{Z})$ and nitrogen 
(N) has been investigated to enhance soil fertility and improve crop production.

\section{Materials and Methods}

The present investigation was carried out at Professor Jayashankar Telangana State Agricultural University (PJTSAU), College Of Agriculture, and Rajendranagar which is located in Ranga Reddy district of Telangana state. The soil required for the present experiment has been collected from B block of student farm, College Of Agriculture, Rajendranagar. The red soil required for the experiment was collected from B-Block of Student farm, College of Agriculture, Rajendranagar. The clods in the soil were broken down and the soil is sieved through 2 mm sieve and was mixed with zeolite as per the treatments and after mixing of zeolite, the pot is filled with soil @ $8 \mathrm{~kg} \mathrm{pot}^{-1}$.

The test crop used in this experiment was maize (DHM 117). 4 levels of Zeolite (0, 2.5, 5, $7.5 \mathrm{t} \mathrm{ha}^{-1}$ i.e., $0,8.93,17.26,26.79 \mathrm{~g} \mathrm{pot}^{-1}$ respectively) and 3 levels of nitrogen (100, $150,200 \mathrm{~kg} \mathrm{~N} \mathrm{ha}^{-1}$ i.e., 357.14, 535.71, 714.28 $\mathrm{mg}$ pot $^{-1}$ respectively) were applied in different treatments which were replicated thrice. Phosphorous $\left(\mathrm{P}_{2} \mathrm{O}_{5}\right)$ and Potassium $\left(\mathrm{K}_{2} \mathrm{O}\right)$ were applied @ 60-60 kg ha ${ }^{-1}$ (24.29 $24.29 \mathrm{mg} \mathrm{pot}^{-1}$ ) uniformly to all the treatments including control $\left(\mathrm{Z}_{0} \mathrm{~N}_{0}\right)$. At the end of the maturity stage, cob from each plant from pot was harvested. Grains were separated from the cobs and grain yield was calculated. The soil collected from B block of student farm, College of Agriculture, Rajendranagar is sieved through $2 \mathrm{~mm}$ sieve and is analyzed for initial physical, physicochemical and chemical properties which are presented in the Table 1.

\section{Plant nutrient analysis}

Plant samples collected at 30, 60, 90 DAS and at harvest were shade dried and kept in the hot air oven at $60^{\circ} \mathrm{C}-80^{\circ} \mathrm{C}$ until constant weight is attained. The dried plant samples were then powdered separately treatment wise and were used for analyzing the nutrient contents.

The nitrogen content in the plant and grain samples was determined by micro Kjeldal distillation (Piper, 1966). For estimation of phosphorous and potassium in plant and grain samples were first digested in diacid mixture $\left(\mathrm{HNO}_{3}\right.$ and $\mathrm{HClO}_{4}$ in 9:4 ratio). In digested extract the phosphorous content is determined by Vanado-Molybdo phosphate yellow colour method in Spectrophotometer at $420 \mathrm{~nm}$ and potassium by Flame photometer as described by Piper (1966).

\section{Nutrient uptake}

The dry matter obtained from each treatment and their respective nutrient contents were used to compute nutrient uptake at 30, 60, 90 DAS and at harvest.

$$
\mathrm{N} / \mathrm{P} / \mathrm{K} \text { Uptake }\left(\mathrm{mg} \mathrm{pot}^{-1}\right)=\frac{\mathrm{N} / \mathrm{P} / \mathrm{K} \text { content }(\%) \mathrm{X} \text { Dry matter }\left(\mathrm{g} \mathrm{pot}^{-1}\right)}{100}
$$

The data recorded from the pot culture experiment was statistically computed by adopting factorial completely randomized design using standard procedures (Rao, 1983). The critical difference was used to evaluate the effects of treatments.

\section{Results and Discussion}

\section{Grain yield}

The grain yield of maize ranged from 14.86 to $46.80 \mathrm{~g} \mathrm{pot}^{-1}$ (Table 2 and Fig. 1). The grain 
yield of maize was significantly improved by application of different combinations of nitrogen and zeolite levels. Among all the treatments, $\mathrm{N}_{200} \mathrm{Z}_{7.5}$ (Nitrogen @ $200 \mathrm{~kg} \mathrm{ha}^{-1}$ +Zeolite @ $\left.7.5 \mathrm{t} \mathrm{ha}^{-1}\right)$ resulted in higher grain yield (46.80 $\mathrm{g} \mathrm{pot}^{-1}$ ) which is on par with $\mathrm{N}_{200} \mathrm{Z}_{5}$ (Nitrogen@200 kg ha ${ }^{-1}+$ Zeolite @ 5 $\mathrm{t} \mathrm{ha}^{-1}$ ) where the grain yield recorded was $45.35 \mathrm{~g} \mathrm{pot}^{-1}$. The lowest grain yield was observed in control (14.86 $\left.\mathrm{g} \mathrm{pot}^{-1}\right)$. Among the three nitrogen levels, $\mathrm{N}_{200}$ produced significantly higher grain yield (42.79 $\mathrm{g} \mathrm{pot}^{-1}$ mean value) compared to other levels of nitrogen. Among four zeolite levels, $Z_{7.5}$ produced higher grain yield (36.62 $\mathrm{g} \mathrm{pot}^{-1}$ ) which is significantly superior over other zeolite levels and control (14.86 $\left.\mathrm{g} \mathrm{pot}^{-1}\right)$.

\section{Nitrogen uptake}

$\mathrm{N}$ uptake was significantly affected by the levels of zeolite and nitrogen at 30, 60, 90 DAS and at harvest (Table 3, 4, 5, 6, 7 respectively). Increasing the zeolite dose have significantly improved $\mathrm{N}$ uptake from 179.30 $\mathrm{mg} \operatorname{pot}^{-1}$ to $231.27 \mathrm{mg} \operatorname{pot}^{-1}$ and nitrogen levels also significantly improved $\mathrm{N}$ uptake. Highest $\mathrm{N}$ uptake was registered in $\mathrm{N}_{200}$ (275.86 mg pot ${ }^{-1}$ ), followed by $\mathrm{N}_{150}(204.50$ $\left.\mathrm{mg} \operatorname{pot}^{-1}\right)$ and $\mathrm{N}_{100}\left(136.30 \mathrm{mg}\right.$ pot $\left.^{-1}\right)$, while the interaction effect of zeolite and nitrogen on $\mathrm{N}$ uptake at 30 DAS was non significant, but it was significant at 60 and 90 DAS, by stover. At $60 \mathrm{DAS},\left(465.37 \mathrm{mg} \mathrm{pot}^{-1}\right)$ and 90 DAS, (1066.06 mg pot ${ }^{-1}$ ) significantly higher $\mathrm{N}$ uptake was recorded with the treatment $\mathrm{Z}_{7.5} \mathrm{~N}_{200}$, followed by $\mathrm{Z}_{5} \mathrm{~N}_{200}\left(442.22 \mathrm{mg}\right.$ pot $^{-1}$, $1015.53 \mathrm{mg} \mathrm{pot}^{-1}$ respectively) while the lowest $\mathrm{N}$ uptake at 60 and 90 DAS was obtained from control (90.17 mg pot ${ }^{-1}, 199.04$ $\mathrm{mg} \operatorname{pot}^{-1}$ respectively). At harvest, the highest $\mathrm{N}$ uptake in grain (425.83 $\mathrm{mg} \mathrm{pot}^{-1}$ ) and stover $\left(278.45 \mathrm{mg}\right.$ pot $\left.^{-1}\right)$ was observed in $\mathrm{Z}_{7.5} \mathrm{~N}_{200}$, which was significantly superior over all other treatments and the lowest $\mathrm{N}$ uptake was found in control (26.91 $\left.\mathrm{mg} \mathrm{pot}^{-1}\right)$.

\section{Phosphorous uptake}

P uptake by maize at 30, 60, 90 DAS and at harvest in grain and stover were represented in table 8, 9, 10, 11, 12 respectively. Higher $\mathrm{P}$ uptake at 30, 60, 90 DAS (75.09, 108.63, $180.88 \mathrm{mg} \mathrm{pot}^{-1}$ respectively) and at harvest (118.53, $92.84 \mathrm{mg} \mathrm{pot}^{-1}$ by grain and stover respectively table 11,12 ) was observed in $\mathrm{Z}_{7.5} \mathrm{~N}_{200}$ treatment, but at 90 DAS, the $\mathrm{P}$ uptake in $\mathrm{Z}_{7.5} \mathrm{~N}_{200}$ treatment was on par with $\mathrm{Z}_{5.0} \mathrm{~N}_{200} \quad\left(173.21 \quad \mathrm{mg}\right.$ pot $\left.^{-1}\right)$ which were significantly superior over rest of the treatments and control $(11.84,14.29,31.57$, $27.23,12.12 \mathrm{mg} \mathrm{pot}^{-1}$ respectively).

\section{Potassium uptake}

Increasing the zeolite dose have significantly improved K uptake by maize from $208.42 \mathrm{mg}$ pot $^{-1}$ in $Z_{0}$ level to $268.05 \mathrm{mg} \mathrm{pot}^{-1}$ and nitrogen levels also significantly improved $\mathrm{K}$ uptake at 30 DAS. The zeolite level, $Z_{7.5}$ (268.05 mg pot ${ }^{-1}$ ) recorded significantly higher $\mathrm{K}$ uptake at 30 DAS compared to all other zeolite levels while the lowest $\mathrm{K}$ uptake was noticed in control (72.11 mg pot ${ }^{-1}$ ). Among the nitrogen levels, highest $\mathrm{K}$ uptake was registered in $\mathrm{N}_{200}$ (320.39 mg pot ${ }^{-1}$ ), followed by $\mathrm{N}_{150}\left(235.79 \mathrm{mg} \mathrm{pot}^{-1}\right)$ while the interaction effect of zeolite and nitrogen on $\mathrm{K}$ uptake at 30 DAS was non significant. K uptake was significantly influenced by both the levels of zeolite and nitrogen at 60, 90 DAS and at harvest (Table 13, 14, 15, 16, 17 respectively). $\mathrm{N}_{200} \mathrm{Z}_{7.5}$ showed highest $\mathrm{K}$ uptake at 60, 90 DAS (549.23, $773.17 \mathrm{mg} \mathrm{pot}^{-}$ ${ }^{1}$ respectively) while the lowest $\mathrm{K}$ uptake was observed in control (112.30, $160.45 \mathrm{mg} \mathrm{pot}^{-1}$ respectively). At harvest, the highest $\mathrm{K}$ uptake in grain (210.59 mg pot ${ }^{-1}$ ) was observed in $\mathrm{Z}_{7.5} \mathrm{~N}_{200}$, which was significantly superior over all other treatments followed by $\mathrm{Z}_{5} \mathrm{~N}_{200}$ (191.97 $\mathrm{mg} \mathrm{pot}^{-1}$ ) while the lowest $\mathrm{K}$ uptake by grain was observed in control $(26.75 \mathrm{mg}$ pot $\left.^{-1}\right)$. In stover highest $K$ uptake was 
observed in $\mathrm{Z}_{7.5} \mathrm{~N}_{200}\left(591.72 \mathrm{mg} \operatorname{pot}^{-1}\right)$ followed by $\mathrm{Z}_{5} \mathrm{~N}_{200}\left(566.98 \mathrm{mg}^{-1} \mathrm{pot}^{-1}\right)$ and the lowest $\mathrm{K}$ uptake was found in control (162.04 $\left.\operatorname{mg} \operatorname{pot}^{-1}\right)$.

Table.1 Properties of the experimental soil (Initial) and zeolite used in the experiment

\begin{tabular}{|c|c|c|c|c|}
\hline \multicolumn{2}{|l|}{ Initial soil properties } & & \\
\hline S. No. & Property & Values & Property & Values \\
\hline 1. & Sand $(\%)$ & 87.36 & 8.Available P $\left(\mathrm{kg} \mathrm{ha}^{-1}\right)$ & 15.48 \\
\hline 2. & Silt $(\%)$ & 4.40 & 9. Available $\mathrm{K}\left(\mathrm{kg} \mathrm{ha}^{-1}\right)$ & 380.66 \\
\hline 3. & Clay $(\%)$ & 8.24 & Zeolite properties & \\
\hline 4. & Soil Texture & Loamy sand & 1. Water Absorption & $\mathbf{9 0 - 1 0 0 \%}$ \\
\hline & $\mathrm{pH}$ & 7.08 & 2.Bulk Density $\left(\mathrm{Mg} \mathrm{m}^{-3}\right)$ & $\mathbf{0 . 3 5 - 0 . 4 5}$ \\
\hline 5 & $\mathrm{EC}\left(\mathrm{dSm}^{-1}\right)$ & 0.45 & $3 . \mathrm{pH}$ & $\mathbf{8 . 0 - 9 . 0}$ \\
\hline 6 & Organic Carbon $(\%)$ & 0.57 & 4. EC $\left(\mathrm{dS} \mathrm{m}^{-1}\right)$ & $\mathbf{5 . 5}$ \\
\hline 7 & Available $\mathrm{N}\left(\mathrm{kg} \mathrm{ha}^{-1}\right)$ & 177.00 & 5. CEC $\left({\mathrm{cmol}\left(\mathrm{p}+\mathrm{kg}^{-1}\right)}^{\mathbf{1 3 0 - 1 3 5}}\right.$ \\
\hline
\end{tabular}

Table.2 Effect of different levels of nitrogen and zeolite on grain yield $\left(\mathrm{g} \mathrm{pot}^{-1}\right)$ of maize

\begin{tabular}{|l|l|l|l|l|l|}
\hline \multirow{2}{*}{ Levels } & \multicolumn{5}{|l|}{ Grain yield of maize $\left(\mathbf{g ~ p o t}^{\mathbf{- 1}}\right)$} \\
\cline { 2 - 6 } & $Z_{0}$ & $Z_{2.5}$ & $Z_{5}$ & $Z_{7.5}$ & Mean (N) \\
\hline $\mathbf{N}_{\mathbf{1 0 0}}$ & 19.50 & 22.87 & 25.35 & 27.20 & $\mathbf{2 3 . 7 3}$ \\
\hline $\mathbf{N}_{\mathbf{1 5 0}}$ & 29.73 & 33.30 & 34.67 & 35.86 & $\mathbf{3 3 . 3 9}$ \\
\hline $\mathbf{N}_{\mathbf{2 0 0}}$ & 38.10 & 40.89 & 45.35 & 46.80 & $\mathbf{4 2 . 7 9}$ \\
\hline Mean $(\mathbf{Z})$ & $\mathbf{2 9 . 1 1}$ & $\mathbf{3 2 . 3 6}$ & $\mathbf{3 5 . 1 2}$ & $\mathbf{3 6 . 6 2}$ & \\
\hline & $\mathbf{S E}(\mathbf{m}) \pm$ & $\mathbf{C D}(\mathbf{0 . 0 5})$ & & $\mathbf{S E}(\mathbf{m}) \pm$ & $\mathbf{C D}(\mathbf{0 . 0 5})$ \\
\hline $\mathbf{N}$ & 0.26 & 0.78 & $\mathrm{~N} \mathrm{X} \mathrm{Z}$ & 0.53 & 1.55 \\
\hline $\mathbf{Z}$ & 0.31 & 0.90 & & & \\
\hline
\end{tabular}

Table. 3 Effect of different levels of nitrogen and zeolite on Nitrogen uptake $\left(\mathrm{mg} \mathrm{pot}^{-1}\right)$ of maize at 30 DAS

\begin{tabular}{|c|c|c|c|c|c|}
\hline \multirow[t]{2}{*}{ Levels } & \multicolumn{5}{|c|}{ Nitrogen uptake $\left(\mathrm{mg} \mathrm{pot}^{-1}\right)$} \\
\hline & $Z_{0}$ & $Z_{2.5}$ & $Z_{5}$ & $Z_{7.5}$ & $\operatorname{Mean}(\mathbf{N})$ \\
\hline $\mathbf{N}_{100}$ & 116.57 & 126.53 & 138.33 & 163.77 & 136.30 \\
\hline$N_{150}$ & 178.50 & 198.13 & 213.23 & 228.13 & 204.50 \\
\hline $\mathbf{N}_{200}$ & 242.83 & 273.03 & 285.67 & 301.92 & 275.86 \\
\hline \multirow[t]{2}{*}{ Mean $(Z)$} & 179.30 & 199.23 & 212.41 & 231.27 & \\
\hline & $\mathrm{SE}(\mathrm{m}) \pm$ & CD (0.05) & & $\mathrm{SE}(\mathrm{m}) \pm$ & CD (0.05) \\
\hline $\mathbf{N}$ & 2.64 & 7.76 & N X Z & 5.29 & NS \\
\hline $\mathbf{Z}$ & 3.05 & 8.97 & & & \\
\hline
\end{tabular}


Table.4 Effect of different levels of nitrogen and zeolite on Nitrogen uptake $\left(\mathrm{mg} \mathrm{pot}^{-1}\right)$ of maize at 60 DAS

\begin{tabular}{|c|c|c|c|c|c|}
\hline \multirow[t]{2}{*}{ Levels } & \multicolumn{5}{|c|}{ Nitrogen uptake $\left(\mathrm{mg} \mathrm{pot}^{-1}\right)$} \\
\hline & $\mathbf{Z}_{0}$ & $Z_{2.5}$ & $\mathbf{Z}_{5}$ & $Z_{7.5}$ & $\operatorname{Mean}(\mathbf{N})$ \\
\hline $\mathbf{N}_{100}$ & 163.67 & 184.63 & 199.43 & 212.73 & 190.12 \\
\hline$N_{150}$ & 241.03 & 267.27 & 311.53 & 337.47 & 289.33 \\
\hline $\mathbf{N}_{200}$ & 364.67 & 402.20 & 442.22 & 465.37 & 418.61 \\
\hline \multirow[t]{2}{*}{ Mean $(Z)$} & 256.46 & 284.70 & 317.73 & 338.52 & \\
\hline & $\mathrm{SE}(\mathrm{m}) \pm$ & CD (0.05) & & $\mathrm{SE}(\mathbf{m}) \pm$ & CD (0.05) \\
\hline $\mathbf{N}$ & 3.04 & 8.93 & $\mathbf{N X Z}$ & 6.09 & 17.87 \\
\hline $\mathbf{Z}$ & 3.51 & 10.32 & & & \\
\hline
\end{tabular}

Table.5 Effect of different levels of nitrogen and zeolite on Nitrogen uptake $\left(\mathrm{mg} \mathrm{pot}^{-1}\right)$ of maize at 90 DAS.

\begin{tabular}{|c|c|c|c|c|c|}
\hline \multirow[t]{2}{*}{ Levels } & \multicolumn{5}{|c|}{ Nitrogen uptake $\left(\mathrm{mg} \mathrm{pot}^{-1}\right)$} \\
\hline & $\mathbf{Z}_{\mathbf{0}}$ & $Z_{2.5}$ & $\mathbf{Z}_{\mathbf{5}}$ & $Z_{7.5}$ & $\operatorname{Mean}(\mathbf{N})$ \\
\hline $\mathbf{N}_{100}$ & 299.96 & 358.89 & 430.19 & 495.74 & 396.19 \\
\hline $\mathbf{N}_{150}$ & 539.74 & 597.81 & 669.29 & 727.69 & 633.63 \\
\hline $\mathbf{N}_{\mathbf{2 0 0}}$ & 783.35 & 861.16 & 1015.53 & 1066.06 & 931.52 \\
\hline \multirow[t]{2}{*}{ Mean $(Z)$} & 541.02 & 605.95 & 705.00 & 763.17 & \\
\hline & $\mathrm{SE}(\mathrm{m}) \pm$ & CD (0.05) & & $\mathrm{SE}(\mathrm{m}) \pm$ & CD (0.05) \\
\hline $\mathbf{N}$ & 5.19 & 15.22 & NXZ & 10.37 & 30.45 \\
\hline $\mathbf{Z}$ & 5.99 & 17.58 & & & \\
\hline
\end{tabular}

Table.6 Effect of different levels of nitrogen and zeolite on Nitrogen uptake (mg pot-1) by maize grain at harvest

\begin{tabular}{|l|l|l|l|l|l|}
\hline \multirow{2}{*}{ Levels } & \multicolumn{5}{|l|}{ Nitrogen uptake $\left(\mathbf{m g ~ p o t}^{-\mathbf{1}}\right)$} \\
\cline { 2 - 6 } & $Z_{0}$ & $Z_{2.5}$ & $Z_{5}$ & $Z_{7.5}$ & Mean (N) \\
\hline $\mathbf{N}_{\mathbf{1 0 0}}$ & 117.61 & 151.67 & 176.67 & 197.61 & $\mathbf{1 6 0 . 8 9}$ \\
\hline $\mathbf{N}_{150}$ & 220.11 & 259.70 & 281.98 & 298.79 & $\mathbf{2 6 5 . 1 4}$ \\
\hline $\mathbf{N}_{\mathbf{2 0 0}}$ & 320.01 & 351.66 & 408.21 & 425.83 & $\mathbf{3 7 6 . 4 3}$ \\
\hline Mean (Z) & $\mathbf{2 1 9 . 2 4}$ & $\mathbf{2 5 4 . 3 4}$ & $\mathbf{2 8 8 . 9 6}$ & $\mathbf{3 0 7 . 4 1}$ & \\
\hline & SE(m) \pm & CD $(\mathbf{0 . 0 5})$ & & SE(m) \pm & CD (0.05) \\
\hline $\mathbf{N}$ & 2.39 & 7.03 & N X Z & 4.79 & 14.06 \\
\hline $\mathbf{Z}$ & 2.76 & 8.12 & & & \\
\hline
\end{tabular}


Table.7 Effect of different levels of nitrogen and zeolite on Nitrogen uptake $\left(\mathrm{mg} \mathrm{pot}^{-1}\right)$ by maize stover at harvest

\begin{tabular}{|c|c|c|c|c|c|}
\hline \multirow[t]{2}{*}{ Levels } & \multicolumn{5}{|c|}{ Nitrogen uptake $\left(\mathrm{mg} \mathrm{pot}^{-1}\right)$} \\
\hline & $\mathrm{Z}_{0}$ & $\mathrm{Z}_{2.5}$ & $\mathrm{Z}_{5}$ & $\mathrm{Z}_{7.5}$ & $\operatorname{Mean}(\mathbf{N})$ \\
\hline $\mathbf{N}_{100}$ & 36.90 & 51.38 & 67.66 & 86.85 & 60.70 \\
\hline$N_{150}$ & 104.11 & 124.60 & 146.65 & 178.80 & 138.54 \\
\hline $\mathbf{N}_{200}$ & 205.07 & 221.74 & 265.05 & 278.45 & 242.58 \\
\hline Mean $(\mathbf{Z})$ & 115.36 & 132.57 & 159.79 & 181.37 & \\
\hline & $\mathrm{SE}(\mathrm{m}) \pm$ & CD (0.05) & & $\mathrm{SE}(\mathrm{m}) \pm$ & CD (0.05) \\
\hline $\mathbf{N}$ & 1.70 & 5.00 & N X Z & 3.41 & 10.00 \\
\hline $\mathbf{Z}$ & 1.97 & 5.77 & & & \\
\hline
\end{tabular}

Table.8 Effect of different levels of nitrogen and zeolite on phosphorous uptake $\left(\mathrm{mg} \mathrm{pot}^{-1}\right)$ by maize at 30 DAS

\begin{tabular}{|l|l|l|l|l|l|}
\hline \multirow{2}{*}{ Levels } & \multicolumn{4}{l}{ P uptake $\left(\mathbf{m g ~ p o t}^{-1}\right)$} \\
\cline { 2 - 6 } & $Z_{0}$ & $Z_{2.5}$ & $Z_{5}$ & $Z_{7.5}$ & Mean (N) \\
\hline $\mathbf{N}_{\mathbf{1 0 0}}$ & 22.94 & 25.45 & 28.15 & 34.25 & $\mathbf{2 7 . 7 0}$ \\
\hline $\mathbf{N}_{\mathbf{1 5 0}}$ & 32.77 & 43.56 & 48.29 & 53.66 & $\mathbf{4 4 . 5 7}$ \\
\hline $\mathbf{N}_{\mathbf{2 0 0}}$ & 46.56 & 66.57 & 69.86 & 75.09 & $\mathbf{6 4 . 5 2}$ \\
\hline Mean (Z) & $\mathbf{3 4 . 0 9}$ & $\mathbf{4 5 . 2 0}$ & $\mathbf{4 8 . 7 6}$ & $\mathbf{5 4 . 3 3}$ & \\
\hline & $\mathbf{S E}(\mathbf{m}) \pm$ & $\mathbf{C D}(\mathbf{0 . 0 5})$ & & $\mathbf{S E}(\mathbf{m}) \pm$ & $\mathbf{C D}(\mathbf{0 . 0 5})$ \\
\hline $\mathbf{N}$ & 0.69 & 2.03 & $\mathrm{~N} \mathrm{X} \mathrm{Z}$ & 1.38 & 4.06 \\
\hline $\mathbf{Z}$ & 0.80 & 2.35 & & & \\
\hline
\end{tabular}

Table.9 Effect of different levels of nitrogen and zeolite on phosphorous uptake $\left(\mathrm{mg} \mathrm{pot}^{-1}\right)$ by maize at 60 DAS

\begin{tabular}{|l|l|l|l|l|l|}
\hline \multirow{2}{*}{ Levels } & \multicolumn{5}{|l|}{ P uptake $\left(\mathbf{m g ~ p o t ~}^{\mathbf{- 1}}\right)$} \\
\cline { 2 - 6 } & $Z_{0}$ & $Z_{2.5}$ & $Z_{5}$ & $Z_{7.5}$ & Mean (N) \\
\hline $\mathbf{N}_{\mathbf{1 0 0}}$ & 27.65 & 30.67 & 33.57 & 40.02 & $\mathbf{3 2 . 9 8}$ \\
\hline $\mathbf{N}_{\mathbf{1 5 0}}$ & 38.09 & 51.99 & 61.18 & 68.74 & $\mathbf{5 5 . 0 0}$ \\
\hline $\mathbf{N}_{\mathbf{2 0 0}}$ & 61.29 & 90.71 & 103.10 & 108.63 & $\mathbf{9 0 . 9 3}$ \\
\hline Mean (Z) & $\mathbf{4 2 . 3 5}$ & $\mathbf{5 7 . 7 9}$ & $\mathbf{6 5 . 9 5}$ & $\mathbf{7 2 . 4 6}$ & \\
\hline & $\mathbf{S E}(\mathbf{m}) \pm$ & $\mathbf{C D}(\mathbf{0 . 0 5})$ & & $\mathbf{S E}(\mathbf{m}) \pm$ & $\mathbf{C D}(\mathbf{0 . 0 5})$ \\
\hline $\mathbf{N}$ & 0.67 & 1.98 & $\mathrm{~N} \mathrm{X} \mathrm{Z}$ & 1.35 & 3.95 \\
\hline $\mathbf{Z}$ & 0.78 & 2.28 & & & \\
\hline \multicolumn{7}{|c|}{$*$ Control -14.29 m pot $^{-1}$} & & \\
\hline
\end{tabular}


Table.10 Effect of different levels of nitrogen and zeolite on phosphorous uptake $\left(\mathrm{mg} \mathrm{pot}^{-1}\right)$ by maize at 90 DAS

\begin{tabular}{|l|l|l|l|l|l|}
\hline \multirow{2}{*}{ Levels } & \multicolumn{4}{|l|}{ P uptake $\left(\mathbf{m g ~ p o t ~}^{\mathbf{- 1}}\right)$} \\
\cline { 2 - 6 } & $Z_{0}$ & $Z_{2.5}$ & $Z_{5}$ & $Z_{7.5}$ & Mean (N) \\
\hline $\mathbf{N}_{\mathbf{1 0 0}}$ & 50.45 & 58.40 & 68.25 & 78.19 & $\mathbf{6 3 . 8 2}$ \\
\hline $\mathbf{N}_{\mathbf{1 5 0}}$ & 79.83 & 96.56 & 113.03 & 122.44 & $\mathbf{1 0 2 . 9 7}$ \\
\hline $\mathbf{N}_{\mathbf{2 0 0}}$ & 121.49 & 146.17 & 173.21 & 180.88 & $\mathbf{1 5 5 . 4 4}$ \\
\hline Mean (Z) & $\mathbf{8 3 . 9 2}$ & $\mathbf{1 0 0 . 3 8}$ & $\mathbf{1 1 8 . 1 6}$ & $\mathbf{1 2 7 . 1 7}$ & \\
\hline & $\mathbf{S E}(\mathbf{m}) \pm$ & $\mathbf{C D}(\mathbf{0 . 0 5})$ & & $\mathbf{S E}(\mathbf{m}) \pm$ & $\mathbf{C D}(\mathbf{0 . 0 5})$ \\
\hline $\mathbf{N}$ & 1.48 & 4.36 & $\mathrm{~N} \mathrm{X} \mathrm{Z}$ & 2.97 & 8.72 \\
\hline $\mathbf{Z}$ & 1.71 & 5.03 & & & \\
\hline
\end{tabular}

Table.11 Effect of different levels of nitrogen and zeolite on phosphorous uptake $\left(\mathrm{mg} \mathrm{pot}^{-1}\right)$ by maize grains

\begin{tabular}{|l|l|l|l|l|l|}
\hline \multirow{2}{*}{ Levels } & \multicolumn{4}{l}{ P uptake $\left(\mathbf{m g ~ p o t ~}^{\mathbf{- 1}}\right)$} \\
\cline { 2 - 6 } & $Z_{0}$ & $Z_{2.5}$ & $Z_{5}$ & $Z_{7.5}$ & Mean (N) \\
\hline $\mathbf{N}_{\mathbf{1 0 0}}$ & 39.65 & 45.00 & 54.13 & 57.02 & $\mathbf{4 8 . 9 5}$ \\
\hline $\mathbf{N}_{\mathbf{1 5 0}}$ & 60.42 & 74.36 & 77.45 & 81.28 & $\mathbf{7 3 . 3 8}$ \\
\hline $\mathbf{N}_{\mathbf{2 0 0}}$ & 83.82 & 99.50 & 111.89 & 118.53 & $\mathbf{1 0 3 . 4 4}$ \\
\hline Mean (Z) & $\mathbf{6 1 . 3 0}$ & $\mathbf{7 2 . 9 5}$ & $\mathbf{8 1 . 1 6}$ & $\mathbf{8 5 . 6 1}$ & \\
\hline & $\mathbf{S E}(\mathbf{m}) \pm$ & $\mathbf{C D}(\mathbf{0 . 0 5})$ & & $\mathbf{S E}(\mathbf{m}) \pm$ & $\mathbf{C D}(\mathbf{0 . 0 5})$ \\
\hline $\mathbf{N}$ & 0.98 & 2.87 & $\mathrm{~N} \mathrm{X} \mathrm{Z}$ & 1.97 & 5.77 \\
\hline $\mathbf{Z}$ & 1.13 & 3.33 & & & \\
\hline
\end{tabular}

Table.12 Effect of different levels of nitrogen and zeolite on phosphorous uptake $\left(\mathrm{mg} \mathrm{pot}^{-1}\right)$ by maize Stover

\begin{tabular}{|c|c|c|c|c|c|}
\hline \multirow[t]{2}{*}{ Levels } & \multicolumn{5}{|c|}{ P uptake (mg pot $\left.{ }^{-1}\right)$} \\
\hline & $\mathrm{Z}_{0}$ & $\mathrm{Z}_{2.5}$ & $\mathrm{Z}_{5}$ & $\mathrm{Z}_{7.5}$ & $\operatorname{Mean}(\mathbf{N})$ \\
\hline $\mathbf{N}_{100}$ & 17.41 & 22.03 & 27.45 & 31.30 & 24.55 \\
\hline $\mathbf{N}_{150}$ & 30.32 & 46.36 & 52.67 & 57.42 & 46.69 \\
\hline $\mathbf{N}_{200}$ & 52.72 & 73.91 & 85.15 & 92.84 & 76.16 \\
\hline \multirow[t]{2}{*}{ Mean (Z) } & 33.48 & 47.43 & 55.09 & 60.52 & \\
\hline & $\mathrm{SE}(\mathrm{m}) \pm$ & CD (0.05) & & $\mathrm{SE}(\mathrm{m}) \pm$ & CD $(0.05)$ \\
\hline $\mathbf{N}$ & 0.96 & 2.45 & NX Z & 1.67 & 4.90 \\
\hline $\mathbf{Z}$ & 0.83 & 2.83 & & & \\
\hline
\end{tabular}


Table.13 Effect of different levels of nitrogen and zeolite on potassium uptake $\left(\mathrm{mg} \mathrm{pot}^{-1}\right)$ by maize at 30 DAS

\begin{tabular}{|c|c|c|c|c|c|}
\hline \multirow[t]{2}{*}{ Levels } & \multicolumn{5}{|c|}{ K uptake (mg pot $\left.{ }^{-1}\right)$} \\
\hline & $\mathrm{Z}_{0}$ & $\mathrm{Z}_{2.5}$ & $\mathrm{Z}_{5}$ & $\mathrm{Z}_{7.5}$ & $\operatorname{Mean}(\mathbf{N})$ \\
\hline $\mathbf{N}_{100}$ & 136.86 & 147.70 & 160.60 & 188.72 & 158.47 \\
\hline $\mathbf{N}_{150}$ & 206.57 & 227.39 & 246.91 & 262.31 & 235.79 \\
\hline $\mathbf{N}_{200}$ & 281.81 & 315.47 & 331.17 & 353.13 & 320.39 \\
\hline \multirow[t]{2}{*}{ Mean $(Z)$} & 208.42 & 230.19 & 246.22 & 268.05 & \\
\hline & $\mathrm{SE}(\mathrm{m}) \pm$ & CD (0.05) & & $\mathrm{SE}(\mathrm{m}) \pm$ & CD (0.05) \\
\hline $\mathbf{N}$ & 2.95 & 8.67 & N X Z & 5.90 & NS \\
\hline $\mathbf{Z}$ & 3.41 & 10.01 & & & \\
\hline
\end{tabular}

Table.14 Effect of different levels of nitrogen and zeolite on potassium uptake $\left(\mathrm{mg} \mathrm{pot}^{-1}\right)$ by maize at 60 DAS

\begin{tabular}{|l|l|l|l|l|l|}
\hline \multirow{2}{*}{ Levels } & \multicolumn{5}{l|}{ K uptake (mg pot $\left.\mathbf{~}^{-\mathbf{1}}\right)$} \\
\cline { 2 - 6 } & $\mathrm{Z}_{0}$ & $\mathrm{Z}_{2.5}$ & $\mathrm{Z}_{5}$ & $\mathrm{Z}_{7.5}$ & Mean (N) \\
\hline $\mathbf{N}_{\mathbf{1 0 0}}$ & 198.94 & 210.38 & 224.07 & 240.59 & $\mathbf{2 1 8 . 5 0}$ \\
\hline $\mathbf{N}_{\mathbf{1 5 0}}$ & 275.28 & 308.40 & 359.47 & 393.06 & $\mathbf{3 3 4 . 0 5}$ \\
\hline $\mathbf{N}_{\mathbf{2 0 0}}$ & 423.81 & 472.49 & 523.58 & 549.77 & $\mathbf{4 9 2 . 4 1}$ \\
\hline Mean (Z) & $\mathbf{2 9 9 . 3 4}$ & $\mathbf{3 3 0 . 4 2}$ & $\mathbf{3 6 9 . 0 4}$ & $\mathbf{3 9 4 . 4 7}$ & \\
\hline & $\mathbf{S E}(\mathbf{m}) \pm$ & $\mathbf{C D}(\mathbf{0 . 0 5})$ & & $\mathbf{S E}(\mathbf{m}) \pm$ & $\mathbf{C D}(\mathbf{0 . 0 5})$ \\
\hline $\mathbf{N}$ & 3.69 & 10.83 & $\mathrm{~N} \mathrm{X} \mathrm{Z}$ & 7.37 & 21.65 \\
\hline $\mathbf{Z}$ & 4.26 & 12.50 & & & \\
\hline
\end{tabular}

Table.15 Effect of different levels of nitrogen and zeolite on potassium uptake $\left(\mathrm{mg} \mathrm{pot}^{-1}\right)$ I by maize at 90 DAS

\begin{tabular}{|c|c|c|c|c|c|}
\hline \multirow[t]{2}{*}{ Levels } & \multicolumn{5}{|c|}{ K uptake (mg pot $\left.{ }^{-1}\right)$} \\
\hline & $\mathbf{Z}_{\mathbf{0}}$ & $\mathbf{Z}_{2.5}$ & $\mathbf{Z}_{5}$ & $\mathbf{Z}_{7.5}$ & $\operatorname{Mean}(\mathbf{N})$ \\
\hline $\mathbf{N}_{100}$ & 240.60 & 274.01 & 320.49 & 366.02 & 300.28 \\
\hline $\mathbf{N}_{150}$ & 400.35 & 446.11 & 492.00 & 532.22 & 467.67 \\
\hline $\mathbf{N}_{200}$ & 567.74 & 619.20 & 735.71 & 773.17 & 673.95 \\
\hline Mean $(Z)$ & 402.90 & 446.44 & 516.07 & 557.13 & \\
\hline & $\mathrm{SE}(\mathrm{m}) \pm$ & CD (0.05) & & $\mathrm{SE}(\mathrm{m}) \pm$ & CD (0.05) \\
\hline $\mathbf{N}$ & 3.53 & 10.35 & $\mathbf{N} X \mathbf{Z}$ & 7.05 & 20.70 \\
\hline $\mathbf{Z}$ & 4.07 & 11.96 & & & \\
\hline
\end{tabular}

*Control - $160.45 \mathrm{mg} \mathrm{pot}^{-1}$ 
Table.16 Effect of different levels of nitrogen and zeolite on potassium uptake $\left(\mathrm{mg} \mathrm{pot}^{-1}\right)$ by maize grain

\begin{tabular}{|c|l|l|l|l|l|}
\hline \multirow{2}{*}{ Levels } & \multicolumn{5}{l}{ K uptake $\left(\mathbf{m g ~ p o t}^{-\mathbf{1}}\right)$} \\
\cline { 2 - 6 } & $\mathrm{Z}_{0}$ & $\mathrm{Z}_{2.5}$ & $\mathrm{Z}_{5}$ & $\mathrm{Z}_{7.5}$ & Mean (N) \\
\hline $\mathbf{N}_{\mathbf{1 0 0}}$ & 38.99 & 53.39 & 63.38 & 73.43 & $\mathbf{5 7 . 3 0}$ \\
\hline $\mathbf{N}_{\mathbf{1 5 0}}$ & 83.28 & 104.40 & 115.55 & 129.08 & $\mathbf{1 0 8 . 0 8}$ \\
\hline $\mathbf{N}_{\mathbf{2 0 0}}$ & 144.82 & 167.66 & 191.97 & 210.59 & $\mathbf{1 7 8 . 7 6}$ \\
\hline Mean (Z) & $\mathbf{8 9 . 0 3}$ & $\mathbf{1 0 8 . 4 8}$ & $\mathbf{1 2 3 . 6 4}$ & $\mathbf{1 3 7 . 7 0}$ & \\
\hline & $\mathbf{S E}(\mathbf{m}) \pm$ & $\mathbf{C D}(\mathbf{0 . 0 5})$ & & $\mathbf{S E}(\mathbf{m}) \pm$ & $\mathbf{C D}(\mathbf{0 . 0 5})$ \\
\hline $\mathbf{N}$ & 1.07 & 3.15 & $\mathrm{~N} \mathrm{X} \mathrm{Z}$ & 2.14 & 6.93 \\
\hline $\mathbf{Z}$ & 1.24 & 3.63 & & & \\
\hline
\end{tabular}

Table.17 Effect of different levels of nitrogen and zeolite on potassium uptake $\left(\mathrm{mg} \mathrm{pot}^{-1}\right)$ by maize stover

\begin{tabular}{|c|c|c|c|c|c|}
\hline \multirow[t]{2}{*}{ Levels } & \multicolumn{5}{|c|}{ K uptake $\left(\mathrm{mg} \mathrm{pot}^{-1}\right)$} \\
\hline & $\mathrm{Z}_{0}$ & $\mathrm{Z}_{2.5}$ & $Z_{5}$ & $Z_{7.5}$ & Mean $(\mathbf{N})$ \\
\hline $\mathbf{N}_{100}$ & 209.50 & 249.63 & 284.58 & 315.84 & 264.89 \\
\hline $\mathbf{N}_{150}$ & 344.75 & 387.15 & 416.33 & 439.87 & 397.03 \\
\hline $\mathbf{N}_{200}$ & 488.28 & 503.00 & 566.98 & 591.72 & $\mathbf{5 3 7 . 5 0}$ \\
\hline \multirow[t]{2}{*}{ Mean $(Z)$} & 347.51 & 379.93 & 422.63 & 449.14 & \\
\hline & SE(m) \pm & CD (0.05) & & $\mathrm{SE}(\mathrm{m}) \pm$ & CD (0.05) \\
\hline $\mathbf{N}$ & 2.82 & 8.29 & N X Z & 5.65 & 16.57 \\
\hline $\mathbf{Z}$ & 3.26 & 9.57 & & & \\
\hline
\end{tabular}

Fig.1 Effect of different levels of nitrogen and zeolite on grain yield $\left(\mathrm{g} \mathrm{pot}^{-1}\right)$ of maize

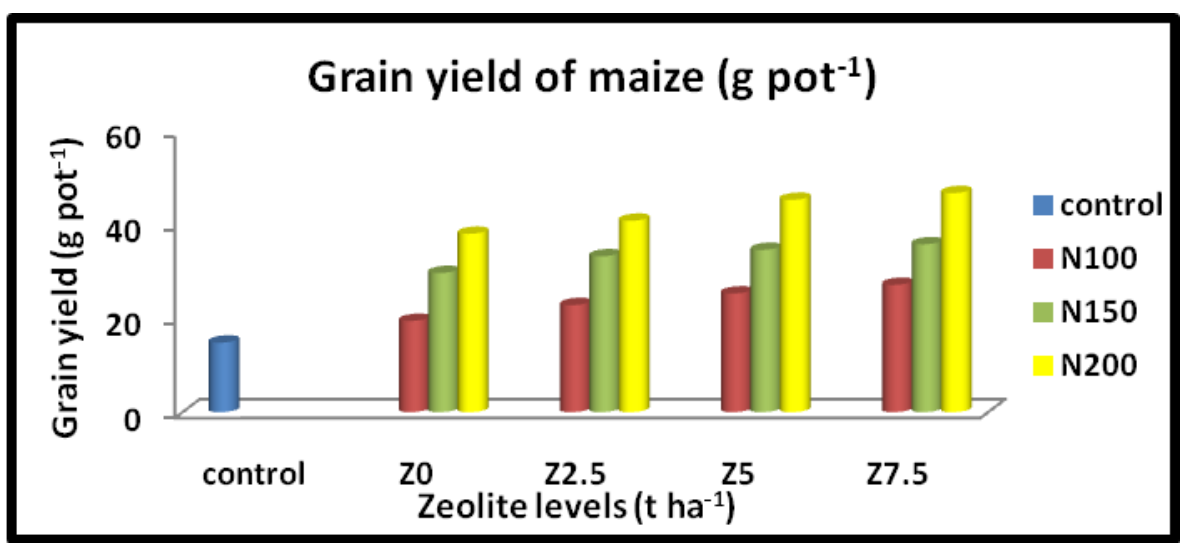

The combined application of nitrogen with zeolite, increased the grain yield of maize due to the slow and controlled release of nitrogen from zeolite and thus making availability of nitrogen throughout the crop growth period which resulted in increased uptake, plant 
height, number of grains per row and test weight, which ultimately lead to increase in the grain yield. These results were comparable to results obtained by Manikandan and Subramanian (2016) where the grain yield of maize in alfisols was increased in zeourea treatment. The enhancement of maize yield with application of zeolite@ $200 \mathrm{~kg} \mathrm{ha}^{-1}$ compared to without application of zeolite was also reported by Weaks et al., (2011).

The slow release pattern of nitrogen by zeolite and reduction of leaching losses which resulted in increased $\mathrm{N}$ availability in the plant root zone might be the responsible factor for enhanced nitrogen uptake. The similar results were obtained by Lija et al., (2014), who reported that combination of zeolite with compound fertilizer enhanced $\mathrm{N}$ uptake in maize. Ahmed et al., (2008) (a) found that zeolite had significantly improved $\mathrm{N}$ uptake in maize and application of higher doses of zeolite along with nitrogen enhanced $\mathrm{N}$ uptake in rice grain and straw (Kavoosi, 2007).

The increase in the $\mathrm{P}$ uptake in maize at different stages with the addition of zeolite may be due to increase in the $\mathrm{P}$ content in the plant tissues and dry matter production. These results were in accordance with the findings of Ahmed et al., (2010) (b) who concluded that irrespective of the treatments, addition of zeolite significantly improved $\mathrm{P}$ uptake in leaves, stems and roots of maize. $\mathrm{P}$ uptake in stems, maize was significantly higher in treatment with compound fertilizer mixed with clinoptilolite zeolite (Rabai et al., (2013).

At 30 DAS there was no significant interaction between zeolite and nitrogen on $\mathrm{K}$ uptake in plants was observed which was in correspondence with the results obtained by Kavoosi (2007), where no significant interaction between zeolite and nitrogen on $\mathrm{K}$ uptake in rice plants was observed. There was a significant influence of both zeolite and nitrogen levels in increasing the $\mathrm{K}$ uptake at 60, 90 DAS and at harvest, due to less leaching of potassium. This is because when zeolites are mixed with soil, they help to retain nutrients from the applied fertilizers in the root zone. These results were in line with the findings of Rabai et al., (2013), who concluded that treatments with clinoptilolite zeolite significantly increased $\mathrm{K}$ uptake in maize stem, roots and leaves. Similar results were also obtained by Ahmed et al., (2010), who found that best $\mathrm{K}$ uptake from all plant tissues of maize from the treatments with zeolite.

\section{References}

Ahmed, O.H., Hussin, A., Ahmad, H.M.H., Rahim, A.A. and Majid, N.M.A. 2008. Enhancing the Urea-N Use Efficiency in Maize (Zea mays) cultivation on Acid soils amended with Zeolite and TSP. The scientific world journal. 8: 394-399.

Ahmed, O.H., Sumalatha, G. and Majid, N.M.A. 2010. Use of zeolite in maize (Zea mays) cultivation on nitrogen, potassium and phosphorus uptake and use efficiency. International Journal of the Physical Sciences. 5(15): 23932401.

He, Z.L., Calvert, D.V., Alva, A.K., Li, Y.C. and Banks, D.J. 2002. Clinoptilolite zeolite and cellulose amendments to reduce ammonia volatilization in a calcareous sandy soil. Plant and Soil. 247: 253-260.

Kavoosi, M. 2007. Effects of Zeolite application on rice yield, nitrogen recovery and nitrogen use efficiency. Communications in Soil Science and Plant Analysis. 38:1-2, 69-76.

Manikandan, A. and Subramanian, K.S. 2016. 
Evaluation of Zeolite based nitrogen Nano-fertilizers on Maize growth, yield and quality on Inceptisols and Alfisols. International Journal of Plant and Soil Science. 9(4): 1-9.

Piper, C.S. 1966. Soil and plant analysis. Hans publishers, Bombay, pp: 137153.

Polat, E., Karaca, M., Demir, H. and Onus, A.N. 2004. Use of natural Zeolite (clinoptilolite) in agriculture. Journal of Fruit and Ornamental Plant Research. 12.

Rabai, K.A., Ahmed, O.H. and Kasim, S. 2013. Use of formulated nitrogen, phosphorus, and potassium compound fertilizer using clinoptilolite zeolite in maize (Zea mays L.) cultivation. Emirates Journal of Food and Agriculture. 25 (9): 713-722.

Ramesh, V., George, J., Jyothi, S.J. and Shibli, S.M.A. 2015. Effect of Zeolites on Soil Quality, Plant Growth and
Nutrient Uptake Efficiency in Sweet Potato (Ipomoea batatas L.). Journal of Root Crops. 41(1): 25-31.

Rao, G.N. 1983. Statistics for Agricultural Science. Oxford and IBH publications, New Delhi.

Szerement, J., Ambrozewicz-Nita, A., Keaziora, K. and Piasek, J. 2014. Use of zeolite in agriculture and environmental protection. A short review.

https://www.researchgate.net/publicati on/268504037_Use_of_zeolite_in_agr iculture_and_environmental_protectio n_A_short_review

Weaks, E.N., Raut, Y., Jahan, H. and Islam, H.R. 2011. Zeolite effects on nitrogen and phosphorus availability in soil. http://scisoc.confex.com/crops/2013a $\mathrm{m} /$ webprogram/Handout/Paper79339/ Zeolite $\% 2 \mathrm{C} \% 20$ Nitrogen\%20and\%20 Phosphoous.pdf

\section{How to cite this article:}

Ravali, CH., K. Jeevan Rao, M. Srilatha and Suresh, K. 2019. Effect of Different Levels of Zeolite and Nitrogen on Grain Yield and Nutrient Uptake of Maize Grown in Red Soil. Int.J.Curr.Microbiol.App.Sci. 8(06): 248-258. doi: https://doi.org/10.20546/ijcmas.2019.806.028 